\title{
Research on the Psychological Activity Process of English Reading
}

\author{
Yu Yang \\ Hainan College of Foreign Studies, Wenchang Hainan, China
}

Keywords: Psychological Activity, English Reading, Learning and Improvement

Abstract. From a psychological point of view, reading is not a passive mechanical process, but a complex psychological process and a proactive thinking process. English reading comprehension is an important topic to improve students' English proficiency and it is also a major research content of psycholinguistics. Psycholinguistics believes that the psychological process of reading includes four stages, namely, the stage of visual perception of words, the stage of sentence structure analysis and interpretation, the stage of discourse comprehension, and the stage of storage and extraction of language information. It can be seen that reading comprehension is not a simple process of deciphering words and passwords, but an extremely complicated psychological process. Therefore, studying the psychological process of English reading comprehension has an important guiding role in doing a good job in English reading teaching. This paper studies and discusses the teaching of English reading class from the perspective of psycholinguistics, and guides the practice of reading class to the practice of reading class. At the same time, it changes some methods of traditional reading class teaching, so that I have a certain results of reading class teaching.

\section{Introduction}

Reading comprehension is an important type of question in all kinds of English proficiency tests. It has a large amount of questions and a large number of points, which directly affects the total score of the test. In practical teaching, although many people recognize the importance of reading teaching, and invest a lot of time and energy, they have spent a lot of thought on improving the quality of teaching, but reading comprehension is still a weakness of students. There are many reasons for this high investment and low output, but the psychological factors are still the most important.

\section{Psychological Process Analysis of Reading Comprehension}

From a psychological point of view, reading is not a passive mechanical process, but a complex psychological process and a proactive thinking process. The famous American psycholinguist Goodman regarded reading as a process of psychological language, pointing out that reading is a psycholinguistic estuary guessing game, which is the process of interaction between thought and language. Reading is a complex process by which the reader reconstructs the information encoded in words as best as possible. 1 Goodman sees this re-constructed behavior as a cyclical process of sampling, prediction, verification, and conviction. Bernhardt believes that foreign language reading has its own characteristics, and should understand the special process of foreign language reading from the perspective of psychology. Cognitiveists represented by Smith encourage readers to exercise subjective initiative and use background knowledge to overcome language barriers. In the study of psycholinguistics, the study of language is extremely important and is a subject that has been explored in academia. The process of understanding the language is the process by which the reader converts his perceived words, words, sentences, paragraphs, etc. into semantics. In this process, readers need to use the various knowledge they have learned, such as the composition of words, words and their meanings, grammar knowledge, text structure, background knowledge, etc., to transform this information into a representation of thinking that is consistent with reality. The process of reading comprehension includes 4

When the reader touches the content of the article, it first maps it to the mind through vision and forms a certain imprint in the brain. This process is the initial stage of the understanding process, and is fully prepared for the following sentence structure and semantic analysis. The visual perception process includes glyph visual perception, word retrieval and recognition. In this process, the reader must make a 
distinctive visual response to the linguistic symbols, ie, glyphs, words, punctuation, etc., and the eye moves from left to right or from top to bottom, and sometimes stops to perceive certain symbols. A word recognition process has been produced. There are two different perspectives on the study of word recognition: one argues that vocabulary extraction is done by activating a set of brain vocabulary representations (logistics). Perceived information such as spelling, speech, part of speech, semantics, etc. are directly entered into the vocabulary storage table. Each lexem e or word is in opposition to a characterization, and once the perceived feature matches the information that characterizes the memory, it achieves the goal of understanding. Another point of view is: The extraction of vocabulary is formed by continuous retrieval of a repository. Perceived information is first converted into a model and then entered into the retrieval system to directly find the information that matches it. Although there are differences between the two modes, they all provide a framework for understanding normal visual vocabulary; vocabulary extraction can be identified by visual-based whole words, or by phonetic rules. The identifiable efficiency is related to the frequency of vocabulary occurrence, context, long-term shortterm memory differences, the intensity of the brain, and the length of the article. Understanding these theories will help students to correctly view the new words in reading comprehension.

Understanding the sentence structure should be examined from both grammatical and meaning perspectives. Both are indispensable and mutually restrictive. In order to understand the meaning of the sentence in the reading process, readers should analyze and explain from the perspective of lexical grammar. The process of interpretation is the process of dividing a sentence into meaningful short paragraph propositions and combining multiple propositions to get the overall meaning. The process of interpretation also includes how to understand the underlying meaning of the sentence. Readers should not only use sentence structure analysis and word meaning search and extraction, but also analyze the relationship between vocabulary and usage, the relationship between words and words, between sentences and sentences, and judge the author's true intention and sentence concealment. Contains meaning. These cognitive abilities involve the reader's cognitive ability, background knowledge, understanding of language and culture, and so on. In pragmatics, speech act theory infers the meaning of words and the meaning of words by analyzing the function of language. The principle of conversational cooperation infers its meaning by analyzing the words that violate the principle of conversational cooperation. In short, interpretation is a complex process. It is a kind of psychological activity of the advanced nervous system. It requires the reader to invoke all verbal and non-verbal (cognitive strategies) methods for information decoding. In this process, the reader fully understands the meaning of the article by visually perceiving the linguistic symbols of the article. Therefore, this is a cognitive processing process associated with linguistic knowledge, personal experience, and the like.

\section{Enlightenment on English Reading Teaching}

A considerable number of students are used to translating all sentences into Chinese when reading, so they are slow to read and the quality of understanding is not high. Smith pointed out that word-by-word or phrase-based reading is extremely harmful, because you may have forgotten the previous meaning before you get the next word, so there is no meaningful connection between words and words. The understanding of the article may become impossible. In short, because they focus their reading on words and narrow the horizon, the reading process for the purpose of obtaining sentences and the overall meaning of the article becomes a process in which students remember the meaning of words or recall the meaning of known words. Understanding is not satisfactory. Therefore, the teacher should adopt the correct scientific teaching steps in the reading class. Over time, the reading speed can be raised.

Students' reading ability is often not due to the lack of vocabulary. Therefore, teachers should teach students as much as possible to memorize words and expand their vocabulary. First of all, the teaching materials with high frequency of occurrence of the same vocabulary should be selected, and relevant background and context-directed teaching should be sought to consolidate the derivative knowledge (prefix and root) of the lecture words, reading memory method, reasoning method, word segmentation, 
synonymy, Anti-sense laws, etc., to expand the vocabulary of students. Second, the reading course is combined with grammar teaching. With the addition of vocabulary, it can't be said that the meaning of the sentence can be understood. Language teaching should teach the students the pronunciation, shape, meaning and usage rules of the language, so as to help students to effectively remember words and expand their vocabulary.

I often hear students complain: Many words in the article are known, but they are readable and unintelligible. The author believes that the main reason for this is that students are not good at interpreting linguistic signs. For example, there is such a sentence, Those who were able to take advantage of the opportunities for success. All the words in this sentence are known to all, but if the reader does not analyze its grammatical structure, it will feel very understanding. difficult. It implies a grammatical phenomenon. Attributive clauses, through grammatical analysis, we know that: The central meaning of a sentence is that capable people can often seize the opportunity of success. Therefore, students should have good habits when they encounter lengthy sentences. First analyze the sentence structure and grammatical structure. This sentence may not be difficult to understand. This requires the teacher to pay attention to the training in the teaching process. The students consciously follow the teacher's guidance when doing the reading exercises.

In teaching, not only to guide students to understand what the article has said, but also to guide students to pay attention to how the author conceived and created the article, that is, to guide students to focus on the article's constitution and organizational structure, language strategy, language logic and language. Means, guide students to reason, analyze, summarize, summarize and understand the content of the article; guide students to understand the author's writing intentions and writing techniques, strengthen students' reasoning of logical relations, and speculate on information content. In teaching, reading and writing can be combined. For example, when teaching writing, we usually pay attention to the use of transitional words, so in reading, students can also pay attention to the application of discourse in discourse. In addition, the use of the topic sentence of the article paragraph should also be noted in the reading. The topic sentence often appears at the beginning, middle or end of the article. By grasping the topic sentence, you can quickly grasp the article's general idea. It can be seen that guiding students to pay attention to the understanding of the composition of the article can achieve a truly effective reading comprehension. Mr. Wang Zuoliang once pointed out: Without knowing the social culture in the language, no one can really master the language. 8 While teaching language knowledge, English teachers should also pay attention to the teaching of target language culture knowledge. Reading an article is a silent process of communication between the reader and the author. The reader lacks knowledge or little knowledge of the author's background knowledge, and it is difficult for readers to enter the author's world and understand the article. An efficient understanding requires the reader to have the ability to relate the author to the background knowledge of the time. This is conducive to understanding and speculating the author's intentions and purposes, reducing the difficulty of communication, and successfully reading. Therefore, students should read more and read more, expand their background knowledge from a large amount of reading materials, establish as many knowledge schemas as possible, and reduce the unfavorable factors brought about by cultural differences to improve reading comprehension.

\section{Teachers Take the Task of Teaching Tasks}

Many students have a large reading volume, but their reading comprehension ability has not been improved. The reason for this phenomenon is that there are three defects in the process of cultivating English reading ability: (1) Teaching ideology and teaching of some teachers The method affects the cultivation of English reading ability. (2) Language knowledge limits the formation of English reading ability. (3) Reading habits, reading psychology, reading methods, and social and cultural background knowledge hindered the development and improvement of English reading ability. - After analyzing and researching this stage of the experience, it is determined that this is the process of psycholinguistic activities, and is the basic stage of the understanding process. In teaching, I guide students to read, read, 
and skim through these reading skills. Skills, fully mobilize students' enthusiasm and speculation ability, have a general understanding of the content of the full text, grasp the main information in the article. At this stage, I think reading speed is an important issue. Students generally have such a mentality. They would rather reduce the reading speed in order to achieve a good understanding. So one of the problems that students need to solve to improve their reading comprehension is to overcome Psychological barriers improve awareness of fast reading. The current solution is that teachers must first clarify to students that the rate of understanding of fast reading is $70 \%$, not $100 \%$ accuracy. In addition, students should be fully aware that: reading speed is reduced, reading level is difficult to improve, and gradually speeding up reading is an important measure that can not be ignored in improving English. There is no speed basis and skill. When reading, there is often a feeling that "the speed is not up to speed", and even the speed of reading cannot be accelerated. The use of skipping, skimming, etc. is an effective measure to get rid of the unsatisfactory speed. Skimming is one of the speed-reading techniques to ensure accurate understanding. The purpose of skipping is to understand the general meaning, basic structure and style of the article and the intention or attitude of the author. Therefore, when I guide students to skip, let students use their eyes. Scan the original text quickly, but grasp the purpose of the article. In other words, skimming requires the reader to read selectively, skipping certain details, in order to grasp the outline of the article, thus speeding up reading.

After research and analysis, it is determined that this is the process of meaning combination of reading psycholinguistic activities, that is, the process of word interpretation and sentence analysis. In teaching, I let students learn to use the prefix, suffix, synonym, antonym, and association method to make speculation. Background knowledge and skills of reading, analyzing language functions, guessing the exact meaning of vocabulary in the article, and inferring the connotation and extension of the word, judging the author's true intentions and the main content of the article, and passing on the article The information, as well as the concealed meaning in the article, are in mind. (3) Reading the third and fourth stages of the psychological model: verification, affirmation or amendment. The third and fourth stages are determined to be the extension process in the reading language mental activity for further study, that is, to use the various information provided in the article to understand the process. The fourth stage, affirmation or revision, is the process of coordination in reading the psychological activities of the language, that is, affirming or correcting the inferences or conclusions made by the reader based on the various information provided by the article. In the process of interrelated and interaction between the third stage and the fourth stage, I made great efforts to train students to learn to use clear topics, find central ideas, topic sentences, important information, make good use of conclusions, and make correct Valuation and other skills and techniques for reading, cultivating them to learn to use the context for a comprehensive understanding - use the content read above to provide clues for later understanding, use the following content to provide feedback on the information obtained before, so that students can correct inappropriate Forecasts, judgments and conclusions.

Reading is a comprehensive process, and the improvement of ability is a gradual process. In any lesson reading teaching, the training and improvement of students' ability cannot be comprehensive. Teachers should put forward practical and feasible time-course goals for all aspects of reading and teaching, and focus on different materials in order to receive better results. Research language understanding is a major subject in experimental psycholinguistics, and the psychological process of reading comprehension is the focus of psycholinguistic research. It is a new field of research on second language reading in recent years. From the perspective of psycholinguistics, I study and discuss the teaching of English reading class, and guide the practice of reading the course and at the same time change some methods of traditional reading class teaching, so that my reading class teaching has a certain Results and results.

\section{Conclusion}

Studying and analyzing the psychological process of reading comprehension can help teachers to choose appropriate teaching methods in foreign language teaching, help them to determine the key points 
and difficulties of teaching, so as to better cultivate students' good reading habits and improve students' reading ability. Of course, the main role of teachers in reading instruction is to encourage students to develop in the correct direction of reading comprehension, and to answer questions in a timely and accurate manner. After all, reading comprehension is mainly done by students themselves through practice. Therefore, only by students carefully comprehending various methods and applying them flexibly to reading practice can we really improve the effect of reading teaching.

\section{Acknowledgment}

Project supported by the Education Department of Hainan Province, project number: Hnjg2018-94.

\section{References}

[1]. Wang Jiankun. English teaching in cultural context [J]. Foreign Languages Journal, 2008(3): 134-136.

[2]. Xu Hongying. Contextual factors in English reading [J]. Journal of Anhui University of Technology (Social Science Edition), 2006(6): 106-108.

[3]. Xu Quan. Language Blocks from the Perspective of Foreign Language Teaching Research: Development and Problems? [J]. Chinese Foreign Languages, 2010(2): 75-79.

[4]. Yao Ximing, Pan Pan. The development of English reading theory research [J]. Foreign Language Teaching, 2004(1): 72-74.

[5]. Yu Xiulian. Experimental Study of Chunk Teaching Method and Improving English Application Ability [J]. Foreign Languages, 2008(3): 54-61. 